\title{
USO DE MEDIDAS NÃO FINANCEIRAS DE DESEMPENHO, ESTRATÉGIA E ORIENTAÇÃO TEMPORAL DE GESTORES DAS 'MELHORES EMPRESAS PARA VOCÊ TRABALHAR'
}

\author{
Andson Braga de Aguiar \\ abraga@usp.br \\ Universidade de São Paulo - São Paulo, SP / Brasil
}

Fábio Frezatti

frezatti@usp.br

Universidade de São Paulo - São Paulo, SP / Brasil

Recebido em 31/08/2013

Aprovado em 11/03/2014

Disponibilizado em 01/04/2014

Avaliado pelo sistema double blind review

Revista Eletrônica de Administração

Editor: Luís Felipe Nascimento

ISSN 1413-2311 (versão on-line)

Editada pela Escola de Administração da Universidade Federal do Rio Grande do Sul.

Periodicidade: Quadrimestral

Sistema requerido: Adobe Acrobat Reader.

\section{RESUMO}

O propósito principal deste estudo é investigar o relacionamento entre o uso de medidas não financeiras de desempenho e orientação temporal dos gestores ao examinar empiricamente predições normativas da contabilidade no que se refere ao relacionamento positivo entre o uso de medidas não financeiras de desempenho e orientação temporal dos gestores. Além disso, este estudo explora o efeito moderador da missão estratégica da empresa sobre o relacionamento entre o uso de medidas não financeiras de desempenho e orientação temporal dos gestores ao examinar se uma missão estratégica de longo prazo intensifica o relacionamento positivo entre medidas não financeiras de desempenho e orientação temporal dos gestores. Portanto, são desenvolvidas hipóteses referentes à associação entre o uso de medidas não financeiras e orientação temporal dos gestores, assim como, se estratégia modera esse relacionamento, tendo por base a literatura contábil relevante sobre o tema. Realiza-se um levantamento em que os questionários são enviados para o endereço eletrônico de uma amostra de gestores de nível intermediário atuando em empresas no Brasil. Utiliza-se a técnica estatística de mínimos quadrados parciais (PLS) e análise de subgrupos para testar as hipóteses de pesquisa. Os resultados indicam a presença de um relacionamento significativo entre uso de medidas não financeiras e orientação temporal dos gestores, mas, contrário às predições normativas e às expectativas deste estudo, uma orientação temporal mais de curto prazo está associada com uma maior importância relativa atribuída a medidas não financeiras de desempenho. Além disso, novamente contrário às expectativas deste estudo, os resultados indicam que a missão estratégica de uma empresa modera esse relacionamento da seguinte maneira: se perseguindo uma estratégia de construir, uso de medidas não financeiras de desempenho está negativamente relacionada com orientação temporal dos gestores. A principal implicação deste estudo é que podem existir certas situações em que o uso de medidas não financeiras de desempenho não contribui para a tomada de decisões de longo

REAd | Porto Alegre - Edição 77 - № 1 - janeiro/abril 2014 - p. 114-139 
prazo. Pesquisa futura poderia explorar essas situações de modo que seja possível obter um entendimento mais amplo dos efeitos de diferentes medidas de desempenho sobre orientação temporal dos gestores.

Palavras-Chave: Estratégia; Medidas de Desempenho; Orientação Temporal dos Gestores.

\title{
USE OF NON-FINANCIAL PERFORMANCE MEASURES, STRATEGY, AND MANAGERIAL TIME ORIENTATION FROM 'BEST COMPANIES TO WORK IN'
}

\begin{abstract}
The main purpose of this study is to investigate the relationship between the use of nonfinancial performance measures and managerial time orientation by empirically examining normative accounting predictions with regard to the positive relationship between the use of non-financial performance measures and managerial time orientation. In addition, this paper explores the moderating effect of firm's strategic mission on the relationship between the use of non-financial performance measures and managerial time orientation by examining whether a long-term strategic mission intensifies the positive relationship between nonfinancial performance measures and managerial time orientation. Therefore, hypotheses are developed concerning the association between the use of non-financial indicators and managerial time orientation, as well as whether strategy moderates this relationship, based on the relevant accounting literature on the theme. A survey is carried out in which questionnaires are sent to the email address of a sample of middle level managers working in companies with business in Brazil. It is applied the statistical technique of partial least squares (PLS) and subgroup analysis to test the research hypotheses. The results indicate the presence of a significant relationship between the use of non-financial performance measures and managerial time orientation, but, contrary to normative predictions and our expectations, a shorter-term orientation is associated with a greater relative importance of non-financial indicators. Moreover, contrary to our expectations either, the results indicate that the firm's strategic mission moderates this relationship as follows: for firms pursuing a build strategy, the use of non-financial measures is negatively related to managerial time orientation. The main implication of this study is that there may be certain situations where the use of nonfinancial performance is does not contribute for long-term decision-making. Future research could explore these situations so that you can get a broader understanding of the effects of different performance measures on managerial time orientation.
\end{abstract}

Keywords: Strategy; Performance Measures; Managerial Time Orientation.

\section{INTRODUÇÃO}

Orientação temporal dos gestores é um aspecto fundamental da tomada de decisão gerencial que reflete trocas compensatórias intertemporais entre efeitos financeiros de curto e de longo prazo. A literatura contábil tem dedicado uma crescente quantidade de atenção aos efeitos de medidas adicionais de desempenho sobre orientação temporal dos gestores (HEMMER, 1996; SLIWKA, 2002; DIKOLLI; VAYSMAN, 2006). A maior parte dessa 
Uso de medidas não financeiras de desempenho, estratégia e orientação temporal de gestores das 'Melhores empresas para você trabalhar'

literatura fundamenta-se no princípio informacional o qual sugere que se uma medida de desempenho tem conteúdo informacional sobre os resultados decorrentes da alocação do esforço gerencial, esse indicador deveria ser adicionado ao sistema de avaliação de desempenho da empresa (HOLMSTROM, 1979). As razões econômicas que explicam a inclusão de medidas não financeiras, além dos indicadores financeiros tradicionais, tais como vendas, custo, lucro e retorno, têm sido amplamente investigadas pela literatura empírica em contabilidade (ITTNER; LARCKER; RAJAN, 1997; BANKER; POTTER; SRINIVASAN, 2000; HASSABELNABY; SAID; WIER, 2005). Adicionalmente, a pesquisa analítica em contabilidade sugere situações em que a inclusão de medidas não-financeiras de desempenho, tais como participação de mercado, volume de vendas, percentual de redução de desperdícios e percentual de falhas de inspeção, é benéfica para a tomada de decisão de longo prazo (DIKOLLI, 2001; SLIWKA, 2002; DIKOLLI; VAYSMAN, 2006). Entretanto, evidências empíricas sobre os prováveis efeitos benéficos de medidas não financeiras sobre orientação temporal dos gestores permanecem escassas; de fato, evidências empíricas recentes, investigando os efeitos diferenciais de indicadores financeiros e não financeiros sobre orientação temporal dos gestores, não têm oferecido suporte às predições normativas em contabilidade (MARGINSON et al., 2010). Portanto, o primeiro objetivo deste estudo é o de investigar o relacionamento entre o uso de medidas não financeiras de desempenho para os propósitos de incentivo e orientação temporal dos gestores.

Além disso, a literatura empírica em contabilidade tem destacado o papel da estratégia nas decisões empresariais de incluir medidas não financeiras de desempenho em seus sistemas de avaliação de desempenho (ITTNER; LARCKER; RAJAN, 1997; SAID; HASSABELNABY; WIER, 2003). Adicionalmente, o efeito moderador da estratégia sobre o relacionamento entre elementos dos sistemas de controle gerencial e desempenho organizacional e gerencial tem sido amplamente reconhecido (LANGFIELD-SMITH, 1997; CHENHALL, 2003). Entretanto, as evidências empíricas existentes não oferecem significativo suporte à expectativa de que estratégia cumpre um papel moderador no relacionamento entre o uso de medidas de desempenho para os propósitos de incentivo e orientação temporal dos gestores (MERCHANT, 1990; VAN DER STEDE, 2000). Portanto, o segundo objetivo deste estudo é o de investigar se a missão estratégica de uma unidade organizacional influencia o relacionamento entre o uso de medidas não financeiras de desempenho e orientação temporal dos gestores. 
A importância em se entender como medidas não financeiras de desempenho e estratégia estão associadas com orientação temporal dos gestores pode ser enumerada em três pontos. Primeiro, medidas financeiras são normalmente 'acusadas' de induzirem os gestores a uma orientação temporal de curto prazo (SLIWKA, 2002; DIKOLLI; VAYSMAN, 2006). Ainda, a fim de mitigar esse efeito negativo, a literatura contábil tem sugerido a inclusão de medidas não financeiras de desempenho (HEMMER, 1996; SLIWKA, 2002; DIKOLLI; VAYSMAN, 2006). A razão para tal sugestão é que, devido medidas não financeiras de desempenho serem percebidas como indicadores de tendência de resultados financeiros futuros, a inclusão de tais indicadores no sistema de avaliação de desempenho deverá conduzir os gestores a pensar nas consequências de longo prazo de suas ações (KAPLAN; NORTON, 1996; ITTNER; LARCKER, 1998). Entretanto, existem poucas evidências empíricas relativas aos prováveis efeitos positivos de medidas não financeiras de desempenho sobre orientação temporal dos gestores. Isso tudo é relevante no discurso de estratégia e manutenção do grupo executivo dentro da organização. Ações inconsistentes demandam energia, tempo e recursos financeiros sem que sejam atingidos os objetivos frequentemente formalizados na organização.

Segundo, é também importante entender como estratégia modera o relacionamento entre medidas não financeiras de desempenho e orientação temporal dos gestores. Apesar da importância que a pesquisa contábil baseada na teoria da contingência atribui ao papel da estratégia como uma variável moderadora para um entendimento mais amplo dos efeitos dos sistemas de controle gerencial sobre comportamento organizacional e individual, pouco se sabe sobre os efeitos moderadores da estratégia sobre o relacionamento entre elementos dos sistemas de controle gerencial e orientação temporal dos gestores. Terceiro, a literatura contábil apenas começa a entender como elementos dos sistemas de controle gerencial afetam orientação temporal dos gestores, sem que haja consenso seja na direção do efeito de diferentes elementos, seja na definição do construto para capturar a orientação temporal dos gestores. Portanto, torna-se relevante o desenvolvimento de pesquisas que procurem identificar o que determina orientação temporal dos gestores.

As contribuições deste artigo podem ser enumeradas em dois pontos. Primeiro, são oferecidas evidências empíricas quanto ao relacionamento entre medidas não financeiras de desempenho e orientação temporal dos gestores ao coletar dados de uma amostra de gestores de nível intermediário. Segundo, são também oferecidas evidências empíricas sobre como a importância relativa de uma missão estratégica de 'construir' influencia o relacionamento 
Uso de medidas não financeiras de desempenho, estratégia e orientação temporal de gestores das 'Melhores empresas para você trabalhar'

entre medidas não financeiras de desempenho e orientação temporal dos gestores. Desse modo, contribuições são oferecidas à pesquisa contábil baseada em contingência que investiga o papel da estratégia em moderar o relacionamento entre os elementos dos sistemas de controle gerencial e o desempenho organizacional e individual.

O restante deste artigo está dividido como segue. Na próxima seção, define-se brevemente orientação temporal dos gestores e sua importância para a literatura contábil. Em seguida, a literatura contábil relevante sobre o tema é revista para o desenvolvimento das hipóteses de pesquisa. Na sequência, o método para coleta de dados é descrito e os principais resultados são apresentados. Na última seção, discutem-se as principais implicações dos resultados desta pesquisa, suas limitações e oportunidades para futuras pesquisas.

\section{ORIENTAÇÃO TEMPORAL DOS GESTORES: DEFINIÇÕES E IMPLICAÇÕES}

Orientação temporal dos gestores reflete as preferências intertemporais dos gestores quando eles têm que tomar decisões com efeitos financeiros sobre o resultado da empresa que ocorrem ao longo do tempo. Em um extremo, têm-se gestores que colocam maior peso a resultados financeiros de curto prazo; em outro extremo, têm-se gestores que atribuem maior importância aos resultados financeiros de longo prazo. Orientação temporal dos gestores pode também ser entendida como a defasagem temporal entre o momento em que os gestores alocam os seus esforços a diferentes tarefas e o momento em que eles recebem as recompensas decorrentes do desempenho nas tarefas (VAN RINSUM, 2002). Embora pareçam similares, essas duas definições são diferentes no sentido de que a primeira está enfatizando os efeitos da alocação do esforço gerencial sobre o desempenho financeiro da empresa, enquanto que a segunda está enfatizando os efeitos sobre a remuneração dos gestores. Consistente com a maior parte da pesquisa normativa contábil define-se orientação temporal dos gestores nesta pesquisa sob o ponto de vista dos efeitos temporais da alocação do esforço gerencial sobre os resultados financeiros da empresa.

Literatura baseada em economia e em psicologia tem dedicado grande atenção ao entendimento dos fatores que influenciam as preferências intertemporais dos indivíduos (METCALFE; MISCHEL, 1999; FREDERICK; LOEWENSTEIN; O’DONOGHUE, 2002; TROPE; LIBERMAN, 2003). A literatura econômica normativa enfatiza a aversão ao risco para predizer que os indivíduos irão preferir concentrar seu consumo no curto prazo (LOEWENSTEIN, 1987). Literatura baseada em economia e em psicologia, que explora aspectos cognitivos do processo de tomada de decisão, destaca o papel de processos mentais

REAd | Porto Alegre - Edição 77 - N 1 - janeiro/abril 2014 - p. 114-139 
nas decisões intertemporais, tais como frequência de informações de acompanhamento, ponto de referência e nível de construção, os quais afetam as preferências intertemporais dos indivíduos (KAHNEMAN; TVERSKY, 1979; BERNATZI; THALER, 1995; TROPE; LIBERMAN, 2003).

Em razão da maior parte da literatura contábil ser baseada em teorias econômicas normativas, especialmente a teoria da agência, o foco dessa literatura tem sido a identificação de quais medidas de desempenho e de quais esquemas de remuneração poderiam mitigar a tendência dos indivíduos de concentrar seu consumo no curto prazo, sendo essa preferência geralmente denominada de miopia gerencial.

A importância em se entender os efeitos de fatores de incentivo sobre orientação temporal dos gestores decorre dos potenciais efeitos negativos que tanto uma orientação temporal de curto prazo quanto uma de longo prazo pode causar sobre o desempenho financeiro atual e futuro de uma empresa: ao consumir tudo hoje, deixa-se nada para ser consumido amanhã; por outro lado, ao poupar tudo para ser consumido amanhã, deixa-se de se ter o que consumir hoje (MATSUMOTO; PRENCHER; RICH, 2000). Portanto, de um lado, ao enfatizar somente resultados financeiros de curto prazo, os gestores podem comprometer a habilidade de uma empresa de criar resultados financeiros futuros; de outro lado, ao enfatizar somente resultados financeiros de longo prazo, os gestores podem enfraquecer a habilidade de uma empresa de criar resultados financeiros de curto prazo. Desse modo, como balancear as trocas compensatórias intertemporais é um dos principais propósitos ao se desenhar sistemas de avaliação de desempenho e de remuneração.

\section{REVISÃO DA LITERATURA E DESENVOLVIMENTO DAS HIPÓTESES}

Esta seção é dividida como segue: primeiro, elabora-se a hipótese referente ao relacionamento entre o uso de medidas não financeiras e orientação temporal dos gestores; segundo, elabora-se a hipótese relativa ao papel moderador da estratégia nesse relacionamento; e, finalmente, apresenta-se o modelo teórico desta pesquisa.

\subsection{Uso de Medidas Não Financeiras e Orientação Temporal dos Gestores}

Geralmente, atribui-se uma orientação gerencial de curto prazo ao uso de medidas financeiras de desempenho para os propósitos de incentivo. Sliwka (2002) menciona que esse efeito ocorre em razão de medidas financeiras de desempenho serem indicadores de ocorrência de muitos aspectos do desempenho organizacional. De modo mais geral, tendo por 
Uso de medidas não financeiras de desempenho, estratégia e orientação temporal de gestores das 'Melhores empresas para você trabalhar'

base a teoria da agência, sugere-se que se somente uma dimensão das tarefas que os gestores têm que desempenhar for mensurada, o valor das demais dimensões dessa tarefa não serão capturados e, como consequência, pouca atenção será dedicada a elas (HOLMSTROM; MILGROM, 1991). Por esse motivo, sugere-se que a inclusão de medidas não-financeiras de desempenho será capaz de capturar outras dimensões das tarefas desempenhadas pelos gestores, as quais poderiam ser negligenciadas se somente medidas financeiras fossem utilizadas (HEMMER, 1996). Medidas financeiras são percebidas como úteis para fornecer informações sobre o nível de esforço relacionado a tarefas que afetam o desempenho financeiro de curto prazo (LAMBERT; LARCKER, 1987; HOSKISSON; HITT; HILL, 1993; DIKOLLI; VAYSMAN, 2006).

Por outro lado, medidas financeiras de desempenho são geralmente criticadas por sua perspectiva de curto prazo, por não serem capazes de fornecer informações tempestivas de acompanhamento relativas aos esforços gerenciais de longo prazo (SAID; HASSABELANABY; WIER, 2003; FARREL; KADOUS; TOWRY, 2008). Tais medidas capturam o nível de esforço exercido para o desempenho de tarefas que melhoram os resultados financeiros de longo prazo somente depois de alguma defasagem temporal (SLIWKA, 2002), não sendo adequadas para encorajar os gestores a pensarem sobre as consequências de longo prazo de seus esforços (DIKOLLI, 2000). Desse modo, medidas financeiras são consideradas como indicadores de ocorrência que não são capazes de fornecer informações sobre a criação de riqueza financeira futura (LAMBERT; LARCKER, 1987; KAPLAN; NORTON, 1996).

Uma vez que medidas financeiras são caracterizadas como refletindo apenas esforços gerenciais de curto prazo e induzindo os gestores a uma orientação temporal de curto prazo, a literatura contábil tem tentado identificar medidas de desempenho adicionais que reflitam os esforços gerenciais de longo prazo a fim de influenciar positivamente a orientação temporal dos gestores. Nesse contexto, medidas não financeiras surgem como uma alternativa, uma vez que são percebidas como capazes de direcionar a atenção dos gestores a atividades que irão afetar também os resultados financeiros de longo prazo (HEMMER, 1996; DIKOLLI; VAYSMAN, 2006). Portanto, como medidas não financeiras são entendidas como sendo relativamente mais tempestivas no fornecimento de informações de ações que afetam resultados financeiros de longo prazo (SAID; HASSABELANABY; WIER, 2003), elas induziriam os gestores na direção de uma orientação temporal mais de longo prazo (KAPLAN; NORTON, 1996; ITTNER; LARCKER, 1998).

REAd | Porto Alegre - Edição 77 - N 1 - janeiro/abril 2014 - p. 114-139 
Apesar da generalização das expectativas normativas de que ao confiar apenas em medidas financeiras, os gestores estarão sendo induzidos a uma orientação temporal mais de curto prazo e, com o intuito de mitigar esse efeito negativo, a inclusão de medidas nãofinanceiras seria capaz de induzir os gestores a uma orientação temporal mais de longo prazo; poucas evidências empíricas têm oferecido suporte para tais expectativas. Van Rinsum e Hartmann (2011), por exemplo, evidenciam que o uso de medidas financeiras de desempenho aumenta o foco dos gestores no curto-prazo, enquanto que medidas não-financeiras seriam capazes de reduzir tal miopia. Por outro lado, Marginson e McAulay (2008) não encontram evidência de que o uso de medidas contábil-financeiras seja a principal causa de uma orientação temporal de curto prazo. Eles destacam o papel de aspectos associados ao indivíduo e organizacionais para explicar a orientação temporal dos gestores de sua amostra. Em outro estudo, Marginson et al. (2010) investigam o uso diferencial de medidas financeiras e não-financeiras de desempenho, tanto o uso diagnóstico quanto o interativo (SIMONS, 2005). Seus resultados indicam que, independentemente do uso específico das medidas financeiras de desempenho, elas não estão associadas a uma orientação temporal de curto prazo; entretanto, quando usadas de modo diagnóstico, medidas não financeiras estão associadas positivamente com uma orientação temporal de curto prazo, mas não quando usadas de maneira interativa. Abernethy, Bouwens e Van Lent (no prelo) também oferecem evidências empíricas de que tanto medidas contábeis quanto medidas não financeiras podem induzir os gestores a uma orientação temporal de mais longo prazo, com as primeiras induzindo os gestores a uma orientação temporal mais de longo prazo do que as medidas não financeiras de desempenho.

Portanto, com o intuito de oferecer evidências adicionais que ajudem no entendimento do relacionamento entre o uso de medidas não financeiras e orientação temporal dos gestores, elabora-se a seguinte hipótese de pesquisa baseada na literatura normativa contábil:

H1: maior importância relativa de medidas não-financeiras de desempenho usadas para os propósitos de incentivo está positivamente relacionada com orientação temporal dos gestores.

\subsection{O Papel Moderador da Estratégia}

Estratégia tem sido amplamente investigada pela pesquisa contábil (LANGFIELDSMITH, 1997; CHENHALL, 2003). Uma das direções de pesquisa dessa literatura contábil é 
Uso de medidas não financeiras de desempenho, estratégia e orientação temporal de gestores das 'Melhores empresas para você trabalhar'

o relacionamento entre tipos estratégicos e sistemas de avaliação de desempenho e remuneração (LANGFIELD-SMITH, 1997; VEEN-DIRKS, 2010), considerando ainda os potenciais efeitos desse relacionamento sobre o desempenho estratégico e financeiro (HO; WU; WU, 2011). Pesquisa contábil baseada em Teoria da Contingência tem também enfatizado o papel moderador da estratégia em afetar o relacionamento entre elementos dos sistemas de controle gerencial e diferentes tipos de resultados (CHENHALL, 2003). Mais especificamente, pesquisa empírica em contabilidade tem investigado se estratégia cumpre um papel moderador no relacionamento entre sistemas de controle gerencial e orientação temporal dos gestores.

A Teoria da Contingência enfatiza a questão de como fatores contingentes afetam o desenho e o funcionamento das organizações (COVALESKI; DIRSMITH; SAMUEL, 1996). Essa perspective teórica passou a ser adotada em pesquisas aplicadas à contabilidade gerencial a partir da metade da década de 1970, tendo por premissa que não há sistemas contábeis universalmente apropriados que sejam igualmente aplicados a todas as organizações em todas as circunstâncias (OTLEY, 1980, p. 413). De modo geral, o principal pressuposto de estudos contingentes é a aceitação de que a adequação entre variáveis estruturais (p. ex., os sistemas de avaliação de desempenho) e variáveis contingentes (p. ex., a estratégia) afeta positivamente o comportamento dos gestores (p. ex. orientação temporal dos gestores) e o desempenho organizacional.

Sob essa perspectiva, Merchant (1990) investiga se diferentes estratégias de crescimento moderam o relacionamento entre pressão para o alcance de metas financeiras e orientação temporal dos gestores, mas não obtém evidências de resultados significantes. Van der Stede (2000) explora o papel de prioridades estratégicas - baixo custo versus diferenciação - como um antecedente do estilo de controle orçamentário que, por sua vez, afetaria orientação temporal dos gestores, em um modelo de mediação. Embora seus resultados tenham identificado um relacionamento negativo entre uma prioridade estratégica de diferenciação e um estilo rígido de controle orçamentário, não se identificou uma associação significativa entre um estilo rígido de controle gerencial e uma orientação temporal de curto prazo; não tendo sido possível validar o efeito da prioridade estratégica sobre orientação temporal dos gestores por meio do estilo de controle gerencial.

Entretanto, nenhum desses dois trabalhos buscou investigar o papel da estratégia no relacionamento entre o uso de diferentes medidas de desempenho para os propósitos de incentivo e orientação temporal dos gestores, uma vez que exploraram o efeito moderador REAd | Porto Alegre - Edição 77 - N 1 - janeiro/abril 2014 - p. 114-139 
(MERCHANT, 1990) e mediador (VAN DER STEDE, 2000) da estratégia no relacionamento entre controles orçamentários, os quais implicam uma confiança em metas contábilfinanceiras (HARTMANN, 2000), e orientação temporal dos gestores.

Desse modo, apesar da ausência de evidências empíricas que examinem o papel que estratégia cumpre no relacionamento entre a importância relativa de diferentes indicadores de desempenho e orientação temporal dos gestores, este estudo se fundamenta em argumentos oriundos da Teoria da Contingência aplicada à pesquisa contábil (CHENHALL, 2003) para predizer que o tipo de missão estratégica irá provavelmente cumprir um papel moderador no relacionamento entre o uso de medidas de desempenho e orientação temporal dos gestores.

Precisamente, evidências empíricas contábeis baseadas em pesquisa contingente sugerem a existência de uma associação (fit) entre uma estratégia de longo prazo, tal como uma missão estratégica de construir (GOVINDARAJAN; GUPTA, 1985; LANGFIELDSMITH, 1997), e sistemas informais de controle gerencial, que confiam mais no uso de medidas contemporâneas de desempenho, tais como medidas não financeiras (CHENHALL, 2003). Como consequência, seria de se esperar que essa associação afetasse de maneira positiva a orientação temporal dos gestores, no sentido de induzí-los a um foco mais de longoprazo. Tendo por base essas expectativas, elabora-se a seguinte hipótese de pesquisa:

H2: o relacionamento positivo entre uma maior importância relativa de medidas nãofinanceiras e orientação temporal dos gestores é maior se a missão estratégica da firma estiver mais próxima de uma estratégia de construir.

\subsection{Modelo da Pesquisa}

A seguir, sumariza-se o modelo desta pesquisa (Figura 1).

Figura 1 - Modelo da Pesquisa

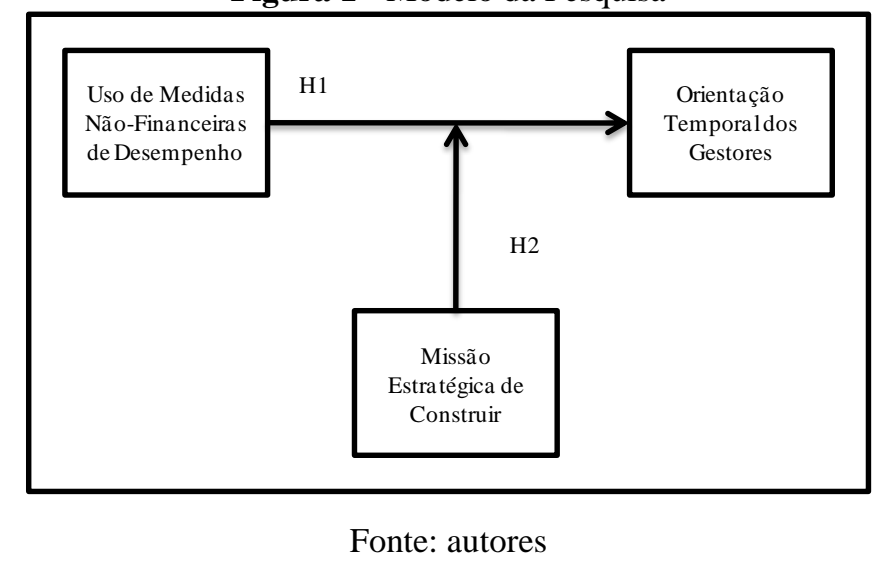

REAd | Porto Alegre - Edição 77 - N 1 - janeiro/abril 2014 - p. 114-139 
Uso de medidas não financeiras de desempenho, estratégia e orientação temporal de gestores das 'Melhores empresas para você trabalhar'

\section{MÉTODO DE PESQUISA}

\subsection{Seleção da Amostra e Taxa de Resposta}

Com o intuito de testar as hipóteses, esta pesquisa desenvolveu um estudo empírico ao coletar dados de gestores de empresas que atuam no Brasil. Foram selecionadas 150 empresas que oferecem aos seus gestores remuneração variável, a partir da publicação 'Melhores Empresas para Você Trabalhar'. Foi realizado um contato inicial com as empresas através do Departamento de Recursos Humanos (RH), com 11 empresas (7,33\% do total) concordando em participar do estudo.

Foi então solicitada ao Departamento de RH das empresas participantes uma lista de gestores que atendessem aos seguintes critérios: (i) tivessem responsabilidade gerenciais, seja como principal executivo de um departamento funcional, tal como vendas, produção etc., seja como o principal executivo de uma divisão, unidade de negócios, centro de resultados ou semelhantes; (ii) trabalhassem em uma área comercial, tal como vendas, produção, pesquisa e desenvolvimento etc.; (iii) recebessem remuneração variável; e (iv) tivessem passado por, pelo menos, um ciclo de avaliação anual de desempenho. $\mathrm{O}$ atendimento a esses critérios identificaria gestores de nível intermediário que tivessem responsabilidade pelo processo de alocação de recursos a diferentes tarefas, cujos desempenhos fossem normalmente avaliados e remunerados com base nesse processo. Foram garantidos o anonimato e a confidencialidade com relação às respostas dos gestores participantes. $\mathrm{O}$ processo de coleta de dados resultou em 129 gestores de nível intermediário que concordaram em participar deste estudo.

Os passos subsequentes do procedimento de coleta de dados seguiram o Método do Desenho Total (Total Design Method - TDM), com o objetivo de aumentar a taxa de resposta (DILLMAN, 2007). Realizou-se um contato inicial com os gestores para convidá-los a participar deste estudo em que teriam que preencher um questionário a ser enviado eletronicamente. Essa mensagem também destacou a importância em contar com a participação desses gestores. Em seguida, foi enviada uma mensagem eletrônica contendo um acesso ao questionário, o qual foi desenvolvido e mantido em um sistema de coleta eletrônica de dados, denominado 'Formsite Web Form Builder'. Como terceiro passo, mensagens eletrônicas de agradecimento foram enviadas com dois os propósitos: primeiro, agradecer aos gestores que já tivessem respondido ao questionário e, segundo, solicitar àqueles gestores que não haviam preenchido o questionário, que o fizessem o mais breve possível. Por fim, foi enviada uma mensagem eletrônica final, incluindo novamente o acesso ao questionário. $\mathrm{O}$ 
objetivo dessa mensagem final foi notificar os gestores cujos questionários ainda não tinham sido identificados e solicitar que eles respondessem.

Ao final do processo de coleta de dados, foram preenchidos 70 questionários, representando uma taxa de resposta de $54,3 \%$, tendo por base o número original de gestores identificados. Desse total, quatro questionários foram descartados para os propósitos de teste das hipóteses, uma vez que os gestores respondentes não atendiam aos critérios estabelecidos para os respondentes. Portanto, 66 questionários foram considerados úteis, correspondendo a uma taxa de resposta de $52,8 \%$.

\subsection{Pré-Teste do Questionário}

O pré-teste do questionário foi conduzido em três fases (DILLMAN, 2007). Na primeira, pesquisadores em contabilidade, com experiência na elaboração de questionários e trabalhos de campo, avaliaram o conteúdo das questões. O Segundo pré-teste foi conduzido por um pesquisador da área de recursos humanos. O propósito dessa segunda fase de pré-teste foi avaliar os termos técnicos do questionário para garantir que eles seriam claramente entendidos em um contexto organizacional. O ultimo pré-teste envolveu um gestor do departamento de logística de uma grande empresa Brasileira cujo perfil se assemelhava ao daquele dos potenciais respondentes; o objetivo foi obter informações sobre dificuldades de preenchimento do questionário e sobre o tempo de preenchimento. A versão final do questionário continha 10 questões, das quais, somente duas eram abertas, divididas em quatro seções e nove páginas. O método TDM foi utilizado com relação à forma de apresentar e à ordem das questões e também em relação à aparência do questionário (DILLMAN, 2007). O Apêndice A apresenta as questões do questionário utilizadas para este estudo.

\subsection{Mensuração das Variáveis}

Tanto quanto possível, procurou-se utilizar instrumentos de mensuração utilizados em estudos anteriores. O modelo de análise considerou o número de fatores identificados em estudos anteriores, de maneira a permitir a comparabilidade.

\subsubsection{Orientação Temporal dos Gestores}

O instrumento para mensurar orientação temporal dos gestores foi originalmente desenvolvido por Jansen, Van Den Bosch e Volberda (2006). Busca-se capturar o nível de importância de se buscar por inovações para clientes e mercados emergentes (exploratory 
Uso de medidas não financeiras de desempenho, estratégia e orientação temporal de gestores das 'Melhores empresas para você trabalhar'

innovation). Essa tarefa envolve realizar esforços e investimentos 'hoje' e esperar algum tempo até que os resultados financeiros sejam afetados. Logo, um maior nível de importância atribuída a essa tarefa irá refletir uma orientação mais de longo prazo. Os respondentes foram solicitados a indicar seu nível de concordância com os seguintes seis itens: (a) minha unidade aceita demanda que vai além dos produtos e dos serviços existentes; (b) minha unidade cria novos produtos e serviços; (c) minha unidade testa novos produtos e serviços em seu mercado; (d) minha unidade vende produtos e serviços que são completamente novos para ela; (e) minha unidade frequentemente explora novas oportunidades em novos mercados; e (f) minha unidade normalmente utiliza novos canais de distribuição. Esses itens representam apenas um fator capturando a tendência de inovações para clientes e mercados emergentes. Uma escala Likert de cinco pontos foi utilizada, parcialmente ancorada, com os seguintes extremos: 1 = discordo totalmente e 5 = concordo totalmente.

\subsubsection{Uso de Medidas Não-Financeiras de Desempenho}

Utilizou-se um instrumento originalmente desenvolvido por Moers (2006) para mensurar o uso de medidas não financeiras de desempenho para os propósitos de incentivo. Esse instrumento avalia a importância percebida de medidas não financeiras de desempenho para avaliar e remunerar os gestores. São utilizados oito itens em que os respondentes tiveram que indicar a importância relativa de medidas financeiras e não financeiras de desempenho geralmente e regularmente utilizadas pelo seu superior para: (a) avaliar seu desempenho; (b) elaborar relatórios periódicos de monitoramento do desempenho; (c) comparar seu desempenho em relação a outros gestores; (d) realizar discussões periódicas com você; (e) determinar o aumento de seu salário; (f) determinar seu bônus anuais; (g) aumentar suas chances de promoção; e (h) aumentar sua autoridade dentro da organização. Esses itens formaram três fatores, cada qual designando um uso específico das medidas de desempenho: (i) propósitos de avaliação (itens 'a,' 'b,' 'c' e 'd'); (ii) propósitos de incentivo monetário (itens 'e' e 'f'); e (iii) propósitos de incentivo não-monetário (itens 'g' e 'h'). Foi utilizada uma escala de cinco pontos, parcialmente ancorada, com os seguintes extremos: 1 = medidas financeiras são muito mais importantes, $3=$ medidas financeiras e não financeiras são igualmente importantes e 5 = medidas não-financeiras são muito mais importantes.

\subsubsection{Estratégia}


Estratégia é mensurada utilizando-se um instrumento usado por Govindarajan e Gupta (1985), o qual avalia a importância relativa das seguintes missões estratégicas: construir, manter, colher e descartar. Os respondentes foram solicitados a indicar a importância relativa, em termos percentuais, dessas estratégias. A soma desses percentuais deveria ser $100 \%$. As quatro estratégias foram representadas pelas seguintes opções: (a) aumentar lucros/vendas e participação de mercado, com interesse em aceitar baixos retornos de médio prazo sobre o investimento, se necessário (construir); (b) manter participação de mercado e obter retorno razoável sobre o investimento (manter); (c) maximizar lucratividade e fluxo de caixa no curto e no médio prazos, com interesse em sacrificar a participação de mercado, se necessário (colher); e (d) preparar para vender ou liquidar (descartar). Além dessas quatro opções, os respondentes poderiam citar outra estratégia. Essa é uma variável ipsative, em que a soma das opções para cada respondente é a mesma (BERGE, 1999), de maneira que a aplicação de uma análise fatorial não é apropriada (DUNLAP; CORNWELL, 1994). Portanto, o percentual atribuído ao item 'a' foi utilizado como medida final para uma missão estratégica de longo prazo.

\subsubsection{Variáveis de Controle}

Quatro variáveis de controle são incluídas neste estudo: empresa, função exercida, nível hierárquico e tempo médio na função. Como mencionado anteriormente, 11 empresas aceitaram participar deste estudo. A Tabela 1 apresenta a função dos respondentes. A Tabela 2 fornece a informação referente ao nível hierárquico dos respondentes. O tempo médio na função foi de 4,5 anos.

Tabela 1 - Função dos Respondentes

\begin{tabular}{l|c|c}
\hline \multicolumn{1}{c}{ Função } & Número & $\mathbf{\%}$ \\
\hline Gestor de Divisão / Unidade & 19 & $28,8 \%$ \\
\hline Produção & 12 & $18,2 \%$ \\
\hline Vendas e Marketing & 27 & $40,9 \%$ \\
\hline Pesquisa \& & 2 & $3,0 \%$ \\
Desenvolvimento & & \\
\hline Outros & 6 & $9,1 \%$ \\
\hline Total Fonte:autores & $\mathbf{6 6}$ & $\mathbf{1 0 0 \%}$ \\
\hline \multicolumn{2}{c}{}
\end{tabular}

Fonte:autores
Tabela 2 - Nível Hierárquico dos Respondentes

\begin{tabular}{l|c|c}
\hline \multicolumn{1}{c|}{ Nível Hierárquico } & Número & $\%$ \\
\hline $1^{\text {o. }}$ Nível Hierárquico & 8 & $12,1 \%$ \\
\hline $2^{\text {o. }}$ Nível Hierárquico & 32 & $48,5 \%$ \\
\hline $3^{\text {o. }}$ Nível Hierárquico & 17 & $25,8 \%$ \\
\hline Outro Nível Hierárquico & 9 & $13,6 \%$ \\
\hline \multicolumn{3}{c}{ Total Fonte:autores }
\end{tabular}

\subsection{Estatística Descritiva}

Em um primeiro exame dos dados, utilizando-se de análise descritiva, apresenta-se a frequência de respostas ao longo da escala Likert (Tabela 3). Para a medida de 'orientação 
Uso de medidas não financeiras de desempenho, estratégia e orientação temporal de gestores das 'Melhores empresas para você trabalhar'

temporal dos gestores', quanto maior a frequência nas escalas ' 4 ' e ' 5 ', mais de longo prazo é a orientação temporal. A maior parte dos respondentes (61\%) apresentou uma alta tendência em direção a uma orientação temporal de longo prazo. Para a frequência do uso de medidas não financeiras para os propósitos de incentivo, percebeu-se que, em média, medidas financeiras de desempenho são relativamente mais importantes para os propósitos de incentivo do que medidas não financeiras de desempenho.

Tabela 3 - Estatística Descritiva

\begin{tabular}{l|c|c|c|c|c|c|c}
\hline \multirow{2}{*}{ Variável } & Unidade de & Intervalo & \multicolumn{5}{c}{ Frequência } \\
\cline { 5 - 8 } & Medida & Teórico & $\mathbf{1}$ & $\mathbf{2}$ & $\mathbf{3}$ & $\mathbf{4}$ & $\mathbf{5}$ \\
\hline Orientação Temporal dos Gestores & Escala Likert & $1-5$ & $10 \%$ & $12 \%$ & $17 \%$ & $34 \%$ & $27 \%$ \\
\hline Medidas Não-Financeiras de Desempenho & Escala Likert & $1-5$ & $16 \%$ & $19 \%$ & $37 \%$ & $14 \%$ & $15 \%$ \\
\hline
\end{tabular}

A variável estratégia adota uma medida ipsativa, de modo que a estatística descritiva para essa variável considera intervalo atual, percentual médio, mediana e desvio padrão em relação a cada uma das opções usadas para mensurar essa variável (Tabela 4). Um maior percentual de uma estratégia de construir indica uma tendência de uma visão mais de longo prazo (GOVINDARAJAN; GUPTA, 1985). O percentual médio atribuído a essa estratégia foi $26 \%$.

Tabela 4 - Estatística Descritiva - Estratégia

\begin{tabular}{|c|c|c|c|c|c|c|}
\hline Estratégia & $\begin{array}{c}\text { Unidade de } \\
\text { Medida } \\
\end{array}$ & $\begin{array}{c}\text { Intervalo } \\
\text { Teórico } \\
\end{array}$ & $\begin{array}{c}\text { Intervalo } \\
\text { Atual } \\
\end{array}$ & $\begin{array}{c}\text { Frequência } \\
\text { Média }\end{array}$ & $\begin{array}{c}\text { Frequência } \\
\text { Mediana }\end{array}$ & $\begin{array}{l}\text { Desvio } \\
\text { Padrão }\end{array}$ \\
\hline Construir & \multirow{5}{*}{$\begin{array}{c}\text { Escala } \\
\text { Ipsativa \% }\end{array}$} & \multirow{5}{*}{$0-100 \%$} & $0-100 \%$ & $26 \%$ & $25 \%$ & $19 \%$ \\
\hline Manter & & & $0-90 \%$ & $34 \%$ & $30 \%$ & $20 \%$ \\
\hline Colher & & & $0-90 \%$ & $31 \%$ & $25 \%$ & $24 \%$ \\
\hline Descartar & & & $0-50 \%$ & $7 \%$ & $0 \%$ & $10 \%$ \\
\hline Outra & & & $0-100 \%$ & $6 \%$ & $0 \%$ & $21 \%$ \\
\hline
\end{tabular}

Fonte: autores

\section{RESULTADOS}

O modelo teórico deste estudo é analisado utilizando-se Mínimos Quadrados Parciais (Partial Least Squares - PLS), que representa uma técnica para estimar Modelos de Equações Estruturais. O pacote estatístico utilizado para desenvolver a análise foi o SmartPLS 2.0.M3 (RINGLE; WENDE; WILL, 2005). A significância dos relacionamentos estruturais foi avaliada através do bootstrapping, uma técnica que estima os coeficientes de correlação entre as variáveis independentes e a variável dependente. Portanto, a fim de avaliar a significância estatística dos relacionamentos preditos em $\mathrm{H} 1$ e $\mathrm{H} 2$, foram avaliados os testes $t$ para a variável independente e para o termo de interação (HARTMANN; MOERS, 1999). 
Para analisar o efeito moderador da estratégia, desenvolveu-se uma análise adicional que foi a correlação de subgrupos. O objetivo dessa análise adicional foi avaliar a forma do efeito interativo (HARTMANN; MOERS, 1999). A análise de subgrupo separa a variável moderadora em dois grupos, um para menores níveis da importância atribuída a uma missão estratégica de construir e outra para maiores níveis de importância atribuída a uma missão estratégica de construir. Em seguida, procede-se à avaliação das correlações entre o uso das medidas não financeiras de desempenho e orientação temporal dos gestores dentro de cada grupo. Os subgrupos foram definidos de acordo com a mediana da variável moderadora.

\subsection{Modelo de Mensuração}

A variável orientação temporal dos gestores representou uma variável de primeira ordem (OTG). A variável uso das medidas não-financeiras para os propósitos de incentivo (NFIN) representou uma variável de segunda ordem, uma vez que incluiu três fatores: (i) propósitos de avaliação (NFIN1); (ii) propósitos de incentivo monetário (NFIN2); e (iii) propósitos de incentivo não-monetário (NFIN3) (MOERS, 2006). A variável 'estratégia' representou um construto unidimensional (ESTRA).

Para todas as variáveis, foram utilizados modelos reflexivos, em que os indicadores representam diferentes manifestações das variáveis latentes (LAW; WONG, 1999). A Tabela 5 apresenta as cargas dos indicadores para as variáveis latentes de primeira ordem, assim como, a confiabilidade composta e a variância média explicada para essas variáveis. A maior parte dos indicadores apresentou correlações acima de 0,7 com suas respectivas variáveis latentes; além disso, as variáveis latentes de primeira ordem apresentaram adequada confiabilidade composta e variância média explicada.

Tabela 5 - Avaliação do Modelo de Mensuração das Variáveis Latentes

\begin{tabular}{llccc}
\hline \multirow{2}{*}{ Variáveis } & \multicolumn{1}{c}{ Indicadores } & Cargas & $\begin{array}{c}\text { Confiabilidade } \\
\text { Composta }\end{array}$ & $\begin{array}{c}\text { Variância Média } \\
\text { Explicada }\end{array}$ \\
\hline \multirow{2}{*}{ OTG } & Inovação Mercados Emergentes I & $0,5738^{2}$ & 0,8932 & 0,5861 \\
& Inovação Mercados Emergentes II & 0,8522 & & \\
& Inovação Mercados Emergentes III & 0,8463 & & \\
& Inovação Mercados Emergentes IV & 0,7975 & & 0,6486 \\
& Inovação Mercados Emergentes V & 0,7700 & & \\
& Inovação Mercados Emergentes VI & 0,7184 & & 0,8805 \\
\hline \multirow{2}{*}{ NFIN1 } & Propósitos de Avaliação I & 0,8610 & & 0,7049 \\
& Propósitos de Avaliação II & 0,8053 & & \\
& Propósitos de Avaliação III & 0,7560 & & \\
& Propósitos de Avaliação IV & 0,7956 & & \\
\hline \multirow{2}{*}{ NFIN2 } & Propósitos de Incentivo Monetário I & 0,9180 & 0,8255 & \\
& Propósitos de Incentivo Monetário II & 0,7530 & & \\
\hline
\end{tabular}

REAd | Porto Alegre - Edição 77 - N 1 - janeiro/abril 2014 - p. 114-139 
Uso de medidas não financeiras de desempenho, estratégia e orientação temporal de gestores das 'Melhores empresas para você trabalhar'

\begin{tabular}{|c|c|c|c|c|}
\hline NFIN3 & $\begin{array}{l}\text { Propósitos de Incentivo Não-Monetário I } \\
\text { Propósitos de Incentivo Não-Monetário II }\end{array}$ & $\begin{array}{l}0,8842 \\
0,8246\end{array}$ & 0,8443 & 0,7309 \\
\hline ESTRA $^{1}$ & Construir & $\mathrm{n} / \mathrm{a}$ & $\mathrm{n} / \mathrm{a}$ & $\mathrm{n} / \mathrm{a}$ \\
\hline
\end{tabular}

A Tabela 6 apresenta as cargas das variáveis de primeira ordem em relação à variável de segunda ordem. Apresenta-se ainda a confiabilidade composta e a variância média explicada. Considerou-se adequada a avaliação do modelo estrutural para as variáveis independentes.

Tabela 6 - Avaliação do Modelo de Mensuração da Variável de Segunda Ordem

\begin{tabular}{clccc}
\hline Variável & Indicadores & Cargas & Confiabilidade Composta & Variância Média Explicada \\
\hline \multirow{2}{*}{ NFIN } & NFIN1 & 0,9173 & 0,8883 & 0,7268 \\
& NFIN2 & 0,8067 & & \\
& NFIN3 & 0,8295 & Fonte: autores
\end{tabular}

Em termos de validade discriminante, a Tabela 7 demonstra que, para todas as variáveis latentes de primeira ordem, mais variância é compartilhada entre a variável latente e seu respectivo bloco de indicadores do que com os indicadores de outras variáveis latentes. Essa análise não incluiu a variável unidimensional STRAT. Em geral, o modelo de mensuração proposto neste estudo provou ser adequado, permitindo a continuidade da análise com a avaliação do modelo estrutural.

Tabela 7 - Avaliação da Validade Discriminante das Variáveis de Primeira Ordem

\begin{tabular}{c|rrrr}
\hline & OTG & NFIN1 & NFIN2 & NFIN3 \\
\hline OTG & 0,7656 & & & \\
NFIN1 & $-0,2667$ & 0,8054 & & \\
NFIN2 & $-0,1967$ & 0,5860 & 0,8396 & 0,8549 \\
NFIN3 & $-0,0977$ & 0,6191 & 0,6151 & 0,8 . \\
\hline
\end{tabular}

Fonte: autores

\subsection{Modelo Estrutural}

A Tabela 8 apresenta, inicialmente, o modelo estrutural das variáveis latentes. Pode se perceber que a variável independente explica seus respectivos indicadores com significância estatística. O principal indicador explicado é o NFIN1. 
Andson Braga de Aguiar \& Fábio Frezatti

Tabela 8 - Avaliação do Modelo Estrutura das Variáveis Latentes

\begin{tabular}{ccc}
\hline Variáveis de $\mathbf{2}^{\mathbf{0}}$ Ordem $\rightarrow$ Variáveis de $\mathbf{1}^{\mathbf{0}}$ Ordem & Coeficientes & Valor $\boldsymbol{t}$ \\
\hline NFIN -> NFIN1 & $0,9159^{*}$ & 35,7295 \\
NFIN -> NFIN2 & $0,8121^{*}$ & 20,5835 \\
NFIN -> NFIN3 & $0,8278^{*}$ & 19,7662 \\
\hline * O relacionamento é significante a um nível de 1\% (bicaudal) & Fonte: autores
\end{tabular}

Em termos de relacionamentos estruturais entre importância relativa de medidas não financeiras e orientação temporal dos gestores, a Tabela 9 apresenta a significância desse relacionamento, assim como, o coeficiente de determinação $\left(\mathrm{R}^{2}\right)$.

Tabela 9 - Avaliação do Efeito Direto de NFIN sobre MTO

\begin{tabular}{lc|c|cc}
\hline & NFIN $\rightarrow$ MTO & Coeficiente & Valor $\boldsymbol{t}$ value & $\mathbf{R}^{\mathbf{2}}$ \\
\hline NFIN -> MTO & * O relacionamento é significante a um nível de 1\% (bicaudal) \\
Fonte: autores & 2,0301 & $16 \%$ \\
\hline \multicolumn{4}{c}{} \\
\end{tabular}

Os resultados demonstraram que o uso das medidas não financeiras de desempenho está significativamente relacionado à orientação temporal dos gestores. Entretanto, contrário às nossas expectativas, um maior nível de importância atribuído às medidas não financeiras de desempenho para os propósitos de incentivo está negativamente associada com orientação temporal dos gestores. Portanto, esses resultados não oferecem suporte para a primeira hipótese de pesquisa (H1) que esperava um relacionamento positivo entre o uso de medidas não financeiras de desempenho e orientação temporal dos gestores. A principal implicação desse resultado é que podem existir certas situações em que o uso de medidas não financeiras de desempenho para os propósitos de incentivo é, na verdade, prejudicial ao invés de benéfico para a tomada de decisão gerencial com foco no longo prazo.

Destaca-se ainda que nenhuma das quatro variáveis de controle incluídas no modelo empresa, função exercida, nível hierárquico e tempo médio na função - apresentaram efeitos estatisticamente significativos sobre orientação temporal dos gestores.

\subsection{Efeito Moderador}

Para analisar o efeito moderador, avaliou-se a significância estatística do coeficiente Beta $(\beta)$ da variável interativa. A Tabela 10 apresenta o coeficiente Beta $(\beta)$ do termo interativo e sua significância estatística (teste $t$ ). 
Uso de medidas não financeiras de desempenho, estratégia e orientação temporal de gestores das 'Melhores empresas para você trabalhar'

Tabela 10 - Avaliação do Termo de Interação

\begin{tabular}{ccc}
\hline & Coeficiente B & Teste $\boldsymbol{t}$ \\
\hline NFIN*STRAT $>$ MTO & $-1,0356$ & 2,4917 \\
\hline & * Significante a um nível de 1\% (bicaudal) \\
Fonte: autores
\end{tabular}

Os resultados sugerem que a importância relativa atribuída a uma missão estratégica de construir modera o relacionamento entre o uso de medidas não financeiras de desempenho e orientação temporal dos gestores. A fim de analisar a forma da interação, realizou-se uma análise de subgrupos (Tabela 11).

Tabela 11 - Análise de Correlação de Subgrupos

\begin{tabular}{c|l|c}
\hline \multicolumn{2}{c|}{\begin{tabular}{c} 
Temporal dos Gestores \\
\multicolumn{3}{c}{ Correlação entre o Uso de Medidas Não-Financeiras de Desempenho e Orientação }
\end{tabular}} & Spearman \\
\hline \multirow{3}{*}{$\begin{array}{c}\text { Missão Estratégica de } \\
\text { Construir }\end{array}$} & Menor Nível de Importância $(\mathrm{n}=40)$ & $-0,053$ \\
& Teste $t$ & 0,744 \\
\cline { 2 - 3 } & Maior Nível de Importância $(\mathrm{n}=26)$ & $-0,433$ \\
& Teste $t$ & $0,027^{*}$ \\
\hline
\end{tabular}

* Correlação é significante a um nível de 5\% (bicaudal)

Fonte: autores

Os resultados sugerem que o efeito moderador da estratégia sobre o relacionamento entre o uso de medidas não financeiras de desempenho e orientação temporal dos gestores ocorre somente quando a missão estratégica da empresa está mais próxima de uma estratégia de construir. A principal implicação desse resultado é que se uma empresa estiver perseguindo uma estratégia de construir, o uso de medidas não financeiras de desempenho para os propósitos de incentivo é, de fato, prejudicial ao invés de benéfica para a orientação temporal dos gestores.

\section{CONCLUSÕES}

Este estudo avaliou e apresentou evidências empíricas relativas ao relacionamento entre o uso de medidas de desempenho para os propósitos de incentivo e orientação temporal dos gestores e relativas ao papel moderador da estratégia. Os resultados indicaram que, contrário às expectativas desta pesquisa e da literatura normativa contábil, uma maior importância atribuída a medidas não financeiras não está positivamente associada com orientação temporal de longo-prazo; ao contrário, os resultados sugerem que uma orientação temporal mais de curto prazo pode estar associada a tais indicadores de desempenho. Embora contrários às predições normativas em contabilidade, estes resultados se somam a outras 
evidências empíricas em contabilidade que também não confirmaram tais predições (MARGINSON et al., 2010; ABERNETHY; BOUWENS; VAN LENT, no prelo), contribuindo para o entendimento do efeito de medidas não-financeiras de desempenho sobre orientação temporal dos gestores. A principal implicação desses resultados é que o uso de medidas não-financeiras de desempenho não contribuem para tomada de decisão de longo prazo.

Os resultados deste estudo também indicaram que estratégia modera o relacionamento entre o uso de medidas não financeiras e orientação temporal dos gestores. Enquanto não se percebeu um efeito moderador para o grupo de respondentes que atribuíram menor importância para uma estratégia de construir, os resultados com base na análise de subgrupos indicaram que, para o grupo de respondentes que atribuíram maior importância para uma estratégia de construir, o uso de medidas não financeiras está negativamente associado com orientação temporal dos gestores. Portanto, diferentes de estudos empíricos anteriores (MERCHANT, 1990; VAN DER STEDE, 2000), os quais não identificaram um efeito moderador e/ou mediador da estratégia, este estudo obteve evidências empíricas de que estratégia pode cumprir um papel moderador no relacionamento entre o uso de medidas de desempenho e orientação temporal dos gestores; contribuindo para a pesquisa contábil baseada em Teoria da Contingência. A principal implicação desses resultados é que unidades organizacionais que perseguem uma estratégia de construir parecem não ser capazes de induzir seus gestores a uma orientação temporal de longo prazo pelo uso de medidas não financeiras de desempenho.

Em geral, este estudo contribui para o entendimento de como um elemento dos sistemas de controle gerencial, os indicadores de desempenho usados para os propósitos de incentivo, está associado com orientação temporal dos gestores ao sugerir que, podem existir situações em que o uso de medidas não financeiras de desempenho conduzirá os gestores a uma orientação temporal de curto prazo.

A principal limitação deste estudo refere-se ao tamanho da amostra. Apesar dos esforços para o aumento da taxa de resposta, a fim de que se obtivesse a quantidade de respondentes considerada adequada, tanto o número limitado de empresas participantes da pesquisa quanto o número de gestores indicados por elas como potenciais respondentes contribuíram para que a amostra final ficasse reduzida. Métodos não paramétricos de análise de dados foram utilizados para tentar superar essa limitação, embora o poder estatístico possa ter sido comprometido, mesmo com a utilização dos referidos métodos. A segunda limitação 
Uso de medidas não financeiras de desempenho, estratégia e orientação temporal de gestores das 'Melhores empresas para você trabalhar'

refere-se ao fato de esta pesquisa utilizar dados cross-sectional, tornando difícil sugerir relacionamentos causais, uma vez que não é possível estabelecer a sequência temporal dos relacionamentos (VAN DER STEDE; YOUNG; CHEN, 2007). Por esse motivo, buscou-se desenvolver esta pesquisa enfatizando a associação entre as variáveis, evitando suposições de causalidade.

Futuros estudos poderiam buscar identificar situações em que o uso de medidas não financeiras estaria negativamente associado com orientação temporal dos gestores, de modo a possibilitar um entendimento mais amplo sobre o tema. Além disso, futuros estudos poderiam utilizar métodos de pesquisa que permitissem a avaliação de relacionamentos causais, tais como estudos longitudinais ou experimentos. Por fim, estudos futuros poderiam desenvolver argumentos teóricos baseados em outras disciplinas, tais como economia e psicologia, para explicar por que o uso de medidas não financeiras de desempenho parece estar negativamente associado com orientação temporal dos gestores.

\section{REFERÊNCIAS}

ABERNETHY, M. A.; BOUWENS, J.; VAN LENT, L. The role of performance measures in the intertemporal choices of business unit managers. Contemporary Accounting Research, New Jersey, no prelo.

BANKER, R. D.; POTTER, G.; SRINIVASAN, D. An empirical investigation of an incentive plan that includes nonfinancial performance measures. The Accounting Review, Sarasota, v. 75 , n. 1 , p. 65-92, 2000.

BERGE, T. A Legitimate Case of Component Analysis of Ipsative Measures, and Partialling the Mean as an Alternative to Ipsatization. Multivariate Behavioral Research, Philadelfia, v. 34, p. 89-102, 1999.

BERNATZI, S.; THALER, R. H. Myopic loss aversion and the equity premium puzzle. The Quarterly Journal of Economics, North Carolina, v. 110, p. 73-92, 1995.

CHENHALL, R. H. Management control systems design within its organizational context: findings from contingency-based research and directions for the future. Accounting, Organizations and Society, Cambridge, v. 28, p. 127-168, 2003.

COVALESKI, M. A.; DIRSMITH, M. W.; SAMUEL, S. Managerial accounting research: the contributions of organizational and sociological theories. Journal of Management Accounting 
Research, Sarasota, v. 8, 1996.

DIKOLLI, S. S. Agent employment horizons and contracting demand for forward-looking performance measures. Journal of Accounting Research, Massachusetts, v. 39, n. 3, p. 481493, 2001.

DIKOLLI, S. S.; VAYSMAN, I. Contracting on the stock price and forward-looking performance. European Accounting Review, London, v. 15, n. 4, p. 445-464, 2006.

DILLMAN, D. A. Mail and internet surveys. New York: John Wiley Professional, 2007. p. 523.

DUNLAP, W. P.; CORNWELL, J. M. Factor analysis of ipsative measures. Multivariate Behavioral Research, Philadelfia, v. 29, n. 1, p. 115-126, 1994.

FARREL, A. M.; KADOUS, K.; TOWRY, K. L. Contracting on contemporaneous vs. forward-looking measures: an experimental investigation. Contemporary Accounting Research, New Jersey, v. 25, n. 3, p. 773-802, 2008.

FREDERICK, S.; LOEWENSTEIN, G.; O'DONOGHUE, T. Time discounting and time preference: a critical review. Journal of Economic Literature, Pennsylvania, v. 40, p. 351-401, 2002.

GOVINDARAJAN, V.; GUPTA, A. K. Linking control systems to business unit strategy: impact on performance. Accounting, Organizations and Society, Cambridge, v. 10, n. 1, p. 5166, 1985.

HARTMANN, F. G. H. The appropriateness of RAPM: toward the further development of theory. Accounting, Organizations and Society, Cambridge, v. 25, p. 451-482, 2000.

HARTMANN, F. G. H.; MOERS, F. Testing contingency hypotheses in budgetary research: an evaluation of the use of moderated regression analysis. Accounting, Organizations and Society, Cambridge, v. 24, p. 291-315, 1999.

HASSABELNABY, H. R.; SAID, A. A.; WIER, B. The retention of nonfinancial performance measures in compensation contracts. Journal of Management Accounting Research, Sarasota, v. 17, p. 23-42, 2005. 
Uso de medidas não financeiras de desempenho, estratégia e orientação temporal de gestores das 'Melhores empresas para você trabalhar'

HEMMER, T. On the design and choice of "modern" management accounting measures. Journal of Management Accounting Research, Sarasota, v. 8, p. 87-116, 1996.

HO, J.; WU, A.; WU, S. Y. Consensus on strategy and its impact on performance. Management Accounting Section Research and Case Conference. Georgia: AAA, 2011.

HOLMSTROM, B. Moral hazard and observability. The Bell Journal of Economics, Califórnia, v. 10, n. 1, p. 74-91, 1979.

HOLMSTROM, B.; MILGROM, P. Multitask principal-agent analyses: incentive contracts, asset ownership, and job design. Journal of Law, Economics, \& Organization, Oxford, v. 7, p. 24-52, 1991.

HOSKISSON, R. E.; HITT, M. A.; HILL, C. W. L. Managerial incentives and investment in R\&D in large multiproduct firms. Organization Science, Maryland, v. 4, n. 2, p. 325-341, 1993.

ITTNER, C. D.; LARCKER, D. F. Are nonfinancial measures leading indicators of financial performance? An analysis of customer satisfaction. Journal of Accounting Research, Massachusetts, v. 36, p. 1-35, 1998.

ITTNER, C. D.; LARCKER, D. F.; RAJAN, M. V. The choice of performance measures in annual bonus contracts. The Accounting Review, Sarasota, v. 72, n. 2, p. 231-255, 1997.

JANSEN, J. J. P.; VAN DEN BOSCH, F. A. J.; VOLBERDA, H. W. Exploratory innovation, exploitative innovation, and performance: effects of organizational antecedents and environmental moderators. Management Science, Maryland, v. 52, n. 11, p. 1661-1674, 2006.

KAHNEMAN, D.; TVERSKY, A. Prospect theory: an analysis of decision under risk. Econometrica, New York, v. 47, n. 2, p. 263-292, 1979.

KAPLAN, R. S.; NORTON, D. P. The Balanced Scorecard. Boston: Harvard University Press, 1996. p. 322.

LAMBERT, R. A.; LARCKER, D. F. An analysis of the use of accounting and market measures of performance in executive compensation contracts. Journal of Accounting Research, Massachusetts, v. 25, p. 85-125, 1987. 
LANGFIELD-SMITH, K. Management control systems and strategy: a critical review. Accounting, Organizations and Society, Cambridge, v. 22, n. 2, p. 207-232, 1997.

LAU, K. S.; WONG, C. Multidimensional constructs in structural equation analysis: an illustration using the perception and job satisfaction constructs. Journal of Management, Califórnia, v. 25, n. 2, p. 143-160, 1999.

LOEWENSTEIN, G. F. Anticipation and the valuation of delayed consumption. The Economic Journal, London, v. 97, p. 666-684, 1987.

MARGINSON, D.; MCAULAY, L. Exploring the debate on short-termism: a theoretical and empirical analysis. Strategic Management Journal, Massachusetts, v. 29, p. 273-292, 2008.

MARGINSON, D. et al. Performance measures and short-termism: an exploratory study. Accounting and Business Research, London, v. 40, n. 4, p. 353-370, 2010.

MATSUMOTO, D.; PEECHER, M. E.; RICH, J. S. Evaluations of Outcome sequences. Organizational Behavior and Human Decision Processes, Califórnia, v. 83, n. 2, p. 331-352, 2000.

MERCHANT, K. A. The effects of financial controls on data manipulation and management myopia. Accounting, Organizations and Society, Cambridge, v. 15, n. 4, p. 297-313, 1990.

METCALFE, J.; MISCHEL, W. A hot/cool system analysis of delay of gratification: dynamics of willpower. Psychological Review, Washington, v. 106, n. 1, p. 3-19, 1999.

MOERS, F. Performance measure properties and delegation. The Accounting Review, Sarasota, v. 81, n. 4, p. 897-924, 2006.

OTLEY, D. T. The contingency theory of management accounting: achievements and prognosis. Accounting, Organizations and Society, Cambridge, v. 5, p. 413-428, 1980.

RINGLE, C. M.; WEND, S.; WILL, A. SmartPLS 2.0 M3 (beta). Germany: University of Hamburg, 2005. Disponível em <http://www..smartpls.de>.

SAID, A. A., HASSABELNABY, H. R.; WIER, B. An empirical investigation of the performance consequences of nonfinancial measures. Journal of Management Accounting Research, Sarasota, v. 15, p. 193-223, 2003.

REAd | Porto Alegre - Edição 77 - N 1 - janeiro/abril 2014 - p. 114-139 
Uso de medidas não financeiras de desempenho, estratégia e orientação temporal de gestores das 'Melhores empresas para você trabalhar'

SLIWKA, D. On the use of nonfinancial performance measures in management compensation. Journal of Economics \& Management Strategy, Illinois, v. 11, n. 3, p. 487$511,2002$.

TROPE, Y.; LIBERMAN, N. Temporal construal. Psychological Review, Washington, v. 110, n. 3, p. 403-421, 2003.

VAN DER STEDE, W. A. The relationship between two consequences of budgetary controls: budgetary slack creation and managerial short-term orientation. Accounting, Organizations and Society, Cambridge, v. 25, p. 609-622, 2000.

VAN DER STEDE, W. A.; YOUNG, S. M.; CHEN, C. X. Doing management accounting survey research. In: CHAPMAN, C.; HOPWOOD, A. G.; SHIELDS, M. D. Handbook of Management Accounting Research. v. 1. Oxford: Elsevier, p. 445-478, 2007.

VAN RINSUM, M. Performance measurement and managerial time orientation. ERIM Ph.D. Series Research in Management, Rotterdam, 2006. 183 p.

VAN RINSUM, M.; HARTMANN, F. The effect of financial and non-financial performance measures on managerial data manipulation and myopia: Analysis of a causal path model. Working Paper, 2011.

VEEN-DIRKS, P. Different uses of performance measures: The evaluation versus reward of production managers. Accounting, Organization and Society, Cambridge, v. 35, p. 141-164, 2010 . 


\section{Apêndice A \\ Mensuração das Variáveis}

\section{Orientação Temporal dos Gestores}

Os gestores foram solicitados a indicar o nível de concordância com as seguintes sentenças em uma escala de cinco pontos. Opção 3 representa uma categoria neutra em que você nem discorda totalmente, nem concorda totalmente. Opções 2 e 4 significam níveis intermediários de nível de discordância e de concordância, respectivamente.

a. Minha unidade aceita demanda que vai além dos produtos e dos serviços existentes;

b. Minha unidade cria novos produtos e serviços;

c. Minha unidade testa novos produtos e serviços em seu mercado;

d. Minha unidade vende produtos e serviços que são completamente novos para ela;

e. Minha unidade frequentemente explora novas oportunidades em novos mercados;

f. Minha unidade normalmente utiliza novos canais de distribuição

\section{Uso de Medidas Não Financeiras de Desempenho}

Os gestores foram solicitados a indicar a importância relativa de medidas financeiras (FIN) e não financeiras (NFIN) de desempenho, explicitamente e regularmente usadas por seu superior, em uma escala de cinco pontos. Opções 1 a 3 têm um grau descendente de importância relativa para FIN e opções 3 a 5 têm um grau de ascendente de importância relativa de NFIN.
a. Avaliar o seu desempenho
b. Elaborar relatórios periódicos de acompanhamento de desempenho
c. Posicionar oficialmente seu desempenho em relação a outros gestores
d. Realizar discussões periódicas com você
e. Determinar o aumento de seu salário
f. Determinar seu bônus anual
g. Aumentar sua chance de promoção
h. Aumentar seu nível de autoridade dentro da organização

\section{Estratégia}

Os gestores foram solicitados a indicar a importância relativa das seguintes estratégias empresariais em sua empresa. (Percentuais devem totalizar 100\%).

a. Aumento nas receitas/vendas e na participação de mercado, havendo interesse em aceitar baixos retornos sobre o investimento no médio-prazo, se necessário

b. Manutenção da participação de mercado e obtenção de razoável retorno sobre o investimento

c. Maximização da lucratividade e do fluxo de caixa no curto- e médio-prazo, havendo interesse em sacrificar participação de mercado, se necessário

d. Preparação para venda ou liquidação

e. Nenhum dos acima. 